\title{
Extracorporeal Shockwaves (ESW) Promote Proliferation and Differentiation of Keratinocytes In vitro-Histology and Immunohistochemistry
}

Vlado Antonic ${ }^{\star *}$, Bernd Hartmann², Sandra Münch², Claudia Belfekroun², Gerald Niedobitek², Beate Petschke ${ }^{3}$, Mark Smith $D^{3}$, Richard Thiele $^{4}$, Wolfgang Schaden ${ }^{5,6}$ and Christian Ottomann ${ }^{7}$

'University of Maryland School of Medicine, Radiation Oncology, Division for Translational Radiation Sciences, Baltimore, Maryland, USA

${ }^{2}$ Unfallkrankenhaus Berlin (UKB), Zentrum für Schwerbrandverletzte, Berlin, Germany

${ }^{3}$ Deutsches Institutfür Zell- und Gewebeersatz (DIZG), Berlin, Germany

${ }^{4}$ Internationals Stosswellenzentrum Berlin (IZB), Berlin, Germany

${ }^{5}$ AUVA-Trauma Center Meidling, Vienna, Austria

${ }^{6}$ Ludwig Boltzmann Institute for Experimental and Clinical Traumatology and Austrian Cluster for Tissue Regeneration, Vienna, Austria

${ }^{7}$ Universitätsklinikum Schleswig-Holstein Campus Lübeck, Sektion Plastische Chirurgie, Intensiveinheit für schwerbrandverletzte, Lübeck, Germany

\begin{abstract}
The objective of presented study was to evaluate in vitro effects of extracorporeal shockwave therapy (ESWT) on the keratinocyte morphology, cytoskeleton and mitotic activity. To determine in vitro effects of ESWT on keratinocytes, we applied 100 pulses with an energy flux density $0.1 \mathrm{~mJ} / \mathrm{mm}^{2}$ and a frequency of $1 \mathrm{~Hz}$ at a distance of $5 \mathrm{~cm}$ between therapy head and culture flask. The treatment parameters were determined in a pilot study. Haematoxylin and Eosin staining, as well as immunohistochemistry for Ki-67, CK5, CK14 and CK10 was performed on the cultured keratinocytes and results were compared between ESW treated group and Controls. No pathomorphological abnormalities or alterations in the cells monolayer could be observed. When assessing histological images of all HE-stained sections of ESW-treated and untreated keratinocytes (control group), no morphological differences could be seen between or within the groups. The proliferation marker Ki-67 was found at a frequency of $14 \%$ for ESW-treated and $10 \%$ for untreated keratinocytes. There were also figures of 95\% (CK5 staining) and 90\% (CK14 staining) for ESW-treated, as well as $85 \%$ (CK5 staining) and $85 \%$ (CK14 staining) for untreated keratinocytes. CK10-positive cells showed a frequency of $24 \%$ in the ESWtreated and $33 \%$ in the untreated cell cultures. Immunohistochemical observations indicated an increased proliferation behaviour following treatment with extracorporeal shockwaves in vitro and maturation of the exposed keratinocytes. Our results suggest an increase in proliferation activity of keratinocytes after in vitro treatment with ESWT. Further investigations of the effects of ESWT on the keratinocyte expression of the chemokines and cytokines are underway.
\end{abstract}

Keywords: Extracorporeal shockwave therapy; Keratinocytes; In vitro; Cytokeratins

\section{Introduction}

Improvement of wound healing is one of the major goals in the scientific community due to high impact on quality of life of patients and their families as well as high costs for the healthcare system associated with treatment of difficult to heal and non-healing wounds. Comorbidities associated with wound healing problems, such as diabetes, smoking, compromised blood perfusion are on the rise. Cell based therapeutic options are showing promising results for improvement of wound healing.

Extracorporeal shockwave therapy (ESWT) is a promising new therapeutic option for treatment of soft tissue wounds [1-3]. It has been successfully used for treatment of full thickness burns, incisional wounds and, importantly, for difficult to heal and non-healing wounds associated with pathologies such as compromised vasculature, diabetes and infections. In skin, ESWT promotes wound healing through suppression of pro-inflammatory pathways, affecting wide array of cytokines and chemokines [4,5]. Additionally, it suppresses infiltration of pro-inflammatory cells (macrophages and neutrophils) in the treated tissue [5]. Due to its effectiveness, non-invasiveness and low risk to benefit ratio, ESWT is becoming more frequently used in the clinics $[1,6]$. The mechanism by which ESWT exerts its beneficial effects has not been fully elucidated and significant efforts in the scientific and medical community are directed towards investigating the cellular and molecular pathways affected by ESWT both in vitro and in vivo. ESWT has significant effects on the rat and human adipocyte-derived stem cells in vivo, and preserves their pluripotency [7] and up regulation of ERK1/2 and cell junction protein [8]. It was shown that ESWT treatment of isolated fibroblasts augments their proliferation and up regulates expression of genes for TGF- $\beta$ and collagen I and II [9].

Keratinocytes are a crucial for successful wound healing. They migrate from the wound edge or other epidermal reservoirs to efficiently re-epithelialize a breach in the integrity of the epidermis. In addition to their importance in the physical filling of the wound bed, they have an important regulatory role in the production of extracellular matrix [10]. Maturation of keratinocytes occurs simultaneously with their migration over the wound bed. Keratinocyte maturation is characterized with pool of cytokeratin, intermediate filament within the cell [11]. During the terminal differentiation of keratinocytes two

*Corresponding author: Vlado Antonic, University of Maryland School of Medicine, Department of Radiation Oncology, Division for Translational Radiation Sciences, Baltimore, Maryland, USA, Tel: 4107060742; E-mail: VAntonic@som.umaryland.edu

Received June 22, 2015; Accepted July 27, 2015; Published August 05, 2015

Citation: Antonic V, Hartmann B, Münch S, Belfekroun C, Niedobitek G, et al (2015) Extracorporeal Shockwaves (ESW) Promote Proliferation and Differentiation of Keratinocytes In vitro-Histology and Immunohistochemistry. J Bioengineer \& Biomedical Sci 5: 161. doi:10.4172/2155-9538.1000161

Copyright: ( 2015 Antonic V, et al. This is an open-access article distributed under the terms of the Creative Commons Attribution License, which permits unrestricted use, distribution, and reproduction in any medium, provided the original author and source are credited. 
Citation: Antonic V, Hartmann B, Münch S, Belfekroun C, Niedobitek G, et al. (2015) Extracorporeal Shockwaves (ESW) Promote Proliferation and Differentiation of Keratinocytes In vitro-Histology and Immunohistochemistry. J Bioengineer \& Biomedical Sci 5: 161. doi:10.4172/21559538.1000161

Page 2 of 5

major classes of proteins, namely tissue-specific cytokeratins on the one hand, and components of the cornified envelope (e.g. loricrin, involucrin) on the other, are differentially expressed and can be used experimentally as differentiation markers [12]. The cytokeratins CK5, CK14 and CK10 are fibrous polypeptides and are important components of the cytoskeleton. They are the most commonly used markers of epithelial differentiation and can be detected in different cell layers in a manner depending on the degree of keratinocyte differentiation. Type II keratin CK5 and type I keratin CK14 form the first cytokeratin pair in keratinocytes whose expression is restricted exclusively to the actively dividing basal cells [13]. During the course of terminal differentiation and keratinization, the keratin expression profile changes in the transition from the proliferative basal cell layer to the post mitotic suprabasal cell layer from the basal cytokeratins CK5/14 to the suprabasal epidermal cytokeratins CK1/10. Several authors used keratinocytes to promote wound healing. Treatment with keratinocytes perform better in acceleration of wound healing $[14,15]$ even when compared to full thickness grafts; There is a critical need to develop culture protocols that augment the proliferative potential of the keratinocytes primary cell culture in order to grow large numbers of cells for recipient-specific wound dressing.

The objective of the present study was to determine the influence of ESWT on the 1) proliferative behaviour of keratinocytes in vitro Ki67 and 2) differentiation of keratinocytes through evaluation of presence of cytokeratins in the primary culture of human keratinocytes.

\section{Materials and Methods}

\section{Keratinocyte isolation and cultivation}

Human split-skin biopsies prepared for the experiments were obtained from routine plastic surgical operations carried out at the Centre for Serious Burns and Plastic Surgery, Emergency Hospital Berlin after approval by the Berlin Ethics Committee and acquisition of informed consent from the patients. The isolation of keratinocytes from biopsies and the application of shockwaves were carried out at the tissue bank of the Emergency Hospital Berlin. For cell cultivation, Dulbecco's modified Eagle medium and DMEM/Ham F12 (1: 1) were used at a ratio of 2:1. These solutions were supplemented (final concentrations given in parentheses) with foetal calf serum, sodium pyruvate $(100 \mathrm{mM})$, L-glutamine $(200 \mathrm{mM})$, gentamycin $(10 \mathrm{mg} /$ $\mathrm{ml})$, cholera toxin, insulin $(10 \mathrm{mg} / \mathrm{ml})$, hydrocortisone $(1 \mathrm{mg} / \mathrm{ml})$ and human EGF. The cultivation of human epidermal keratinocytes was carried out in co-culture with a feeder layer. The keratinocytes were adjusted to a concentration of one million cells $/ \mathrm{ml}$ prior to seeding. Twenty $\mathrm{ml}$ of keratinocyte medium was added to T75 cell culture flasks $\left(75 \mathrm{~cm}^{2}\right)$, which were then inoculated with the keratinocytes at a concentration of 13,500 cells per $\mathrm{cm}^{2}$ and feeder layer was inoculated at 25,000 cells per $\mathrm{cm}^{2}$. A subculture of the adherent cells was made after $80 \%$ confluence was reached between 7 and 10 days after seeding of the keratinocyte culture. After rinsing the cell culture flasks with $10 \mathrm{ml}$ of PBS, the cell layer was incubated for 7 minutes with $5 \mathrm{ml}$ of trypsin/EDTA solution $(0.125 \% / 0.04 \%)$ at $37^{\circ} \mathrm{C}$ in an incubator. After 7 min centrifugation of the cell suspension at $1,000 \mathrm{rpm}$ (Megafuge ${ }^{\circ}$, Heraeus), the supernatant was carefully aspirated and the cell pellet was then resuspended in $5 \mathrm{ml}$ of fresh keratinocyte medium. After the cell number and viability were determined in a Neubauer counting chamber, the subculture was seeded at $32,000-40,000$ cells $/ \mathrm{cm}^{2}$ in T25 cell culture flasks $\left(25 \mathrm{~cm}^{2}\right)$ before they were incubated under standard conditions.

\section{Shock-wave device and ESWT application}

The Ortho Wave $180 c^{\circ}$ device produces electrohydraulic shockwaves in the modified water bath at constant temperature of $37^{\circ} \mathrm{C}$. The separate set of experiments was carried out to test several doses, frequencies and distances in order to find the optimal treatment parameters. Results showed that starting Energy Density Flux (EFD) $=0.1 \mathrm{~mJ} / \mathrm{mm}^{2}$, position of the flasks at $50 \mathrm{~mm}$ distance from the applicator (actual EFD of $0.04 \mathrm{~mJ} / \mathrm{mm}^{2}$ was determined at this position of the flask) and at frequency of $1 \mathrm{~Hz}$ was found to be the most adequate for inducing proliferation and avoiding damage to the keratinocytes. All the ESWT exposure was conducted in the modified heated water bath at constant temperature of $37^{\circ} \mathrm{C}$ (Figures 1A and 1B) filled with $2.500 \mathrm{ml}$ preheated water. The cell culture flasks were filled with 59 $\mathrm{ml}$ of keratinocyte medium and then positioned at $50 \mathrm{~mm}$ from the applicator using removable clamp with the cultured side perpendicular to the shock wave generator. After shock wave treatment, the excess medium in the cell culture flasks was discarded and the keratinocytes were then cultured further under standard conditions. The experiments were carried out in triplicate, while the respective control group were carried out in quadruplicate

\section{Microscopic evaluation}

The assessment of the cultured keratinocyte proliferation and differentiation behaviour was performed using light microscopy of histologically and Immunohistochemically (ICH) stained paraffin sections. Due to the high water content of the cells (about 70\%), and the low presence of high contrast structures, the preparations were stained for the bright field microscopy only after previous fixing and preparation of the paraffin sections. The stains used have varying affinities for the different cell components so that cell structures could easily be differentiated by light microscopy. To fix the cultured keratinocytes, the cell lines were detached and spun down 7 days after shockwave application and the resulting cell pellet was overlaid for subsequent histological preparation with $96 \%$ ethanol. After 10 minutes of centrifugation at 3,000 rpm, the precipitated cell pellets were fixed overnight in $4 \%$ formaldehyde within embedding cassettes. For light microscopic examination of keratinocytes $4 \mu \mathrm{m}$ sections were prepared. For morphological evaluation of keratinocytes, haematoxylin and eosin (HE) staining was carried out on a routine basis.

\section{Immunohistochemistry Ki-67, CK5, CK14, CK10}

ICH staining of the paraffin sections was carried out for Ki-67, CK5, CK14 and CK10 to illustrate the proliferation and differentiation behaviour of the keratinocytes. Ki-67 is preferentially expressed in cells during all active phases of the cell cycle (G1, S, G2 and M-phase) and

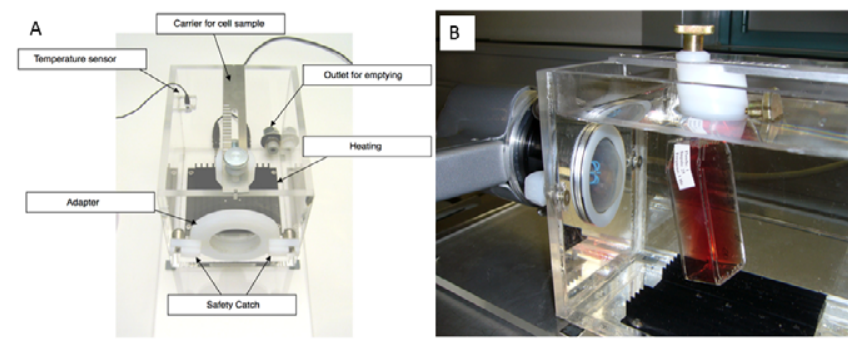

Figure 1: A. Schematic representation of the modified water bath for the use with Ortho Wave ${ }^{\circledR} 180 \mathrm{c}$ electrohydraulic shockwave generator head. B. Image of the experimental setup with position of the cell culture flask in the water bath during ESWT exposure. 
Citation: Antonic V, Hartmann B, Münch S, Belfekroun C, Niedobitek G, et al. (2015) Extracorporeal Shockwaves (ESW) Promote Proliferation and Differentiation of Keratinocytes In vitro-Histology and Immunohistochemistry. J Bioengineer \& Biomedical Sci 5: 161. doi:10.4172/21559538.1000161

serves as such as a universal proliferation marker in ICH [16,17]. In resting cells (G0-phase), however, no Ki-67 expression takes place [18]. All monoclonal antibodies were diluted prior to immunostaining with the ready-to-use DAKO RealTM Antibody Diluent to ensure a specific detection of the antigens without background staining. The staining of cytokeratins and the proliferation marker Ki-67 in the keratinocytes was performed simultaneously in the fully automated ICH staining system Vision Biosystems BondTM. Pre-treatment of paraffin sections was necessary prior to IHC procedure for the retrieval of the epitopes of the antigens of interest.

The quantitative analysis was performed by counting positively stained cells in 5 different fields of view at 200X magnification. The percentage of positively stained cells was then determined in triplicates and compared with that measured for the control group. The intensity of the stained precipitates was qualitatively assessed using following score: $+=$ mild, $++=$ average, $+++=$ strong.

All ICH stained paraffin sections were assessed by direct light microscopy using a Zeiss Axioskop 2 Plus microscope and photographed using the Axio Vision40 LE software at 200X magnification with an AxioCam MRc device before they were analysed using the ImageJ software. Image analysis was performed by the personnel blinded for the study.

\section{Results}

\section{Haematoxylin and eosin}

Light microscopic evaluation of the keratinocyte monolayer before and after the ESWT showed no adverse effect of the shockwaves with optimized parameters $\left(E F D=0.1 \mathrm{~mJ} / \mathrm{mm}^{2}\right.$, distance $50 \mathrm{~mm}$ and frequency $1 \mathrm{~Hz}$ ). The $\mathrm{HE}$ staining of all slides revealed keratinocytes with a round-oval to a flattened and polygonal morphology (Figure 2). The cytoplasm stained intensively with a red-orange colour. The nuclei appeared oval, partially flattened and with a weakly blue colouration, some with distinct nucleoli. There were cells of roundish-oval morphology, corresponding to the appearance of basal cells; however, there were also cells of a polygonal phenotype that corresponded to the morphology of differentiated cells. The nuclei all appeared normal and uniform. Abnormal morphology or alterations in the cells were not observed, either between cells or nor when compared to other groups. There were no morphological differences found upon consideration of photomicrographs of HE-stained sections from all the ESWT and untreated keratinocytes, either within or between the groups (Figure 2).

\section{Immunohistochemistry}

Ki-67 positively stained keratinocyte nuclei clearly differed from the negative, mitotically inactiveblue stained nuclei. The proliferation marker Ki-67 was found at a frequency of $14 \%$ amongst the ESWT and

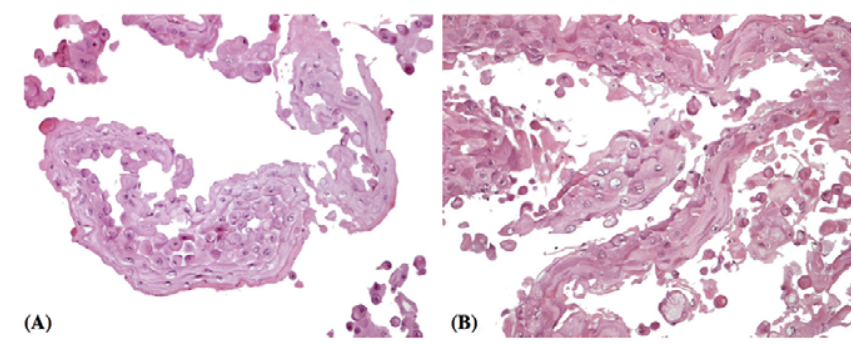

Figure 2: A.Representative image of the $\mathrm{H}$ and $\mathrm{E}$ stained keratinocyte sections in ESWT group. B. Untreated control group.
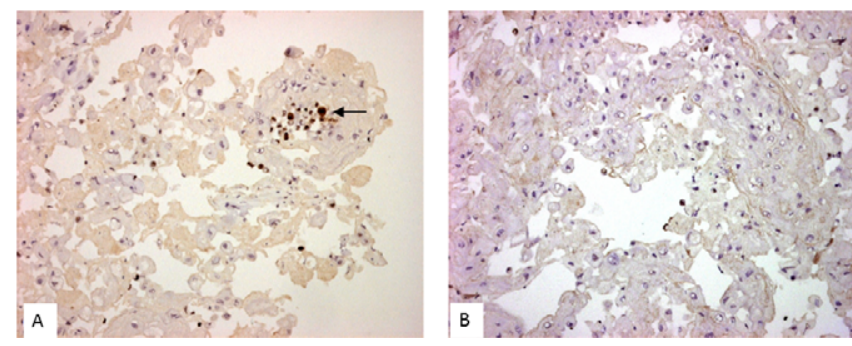

Figure 3: A. Treatment of cells with ESWT induced increase proliferation in keratinocytes. B.Representative image of $\mathrm{IHC}$ staining of $\mathrm{Ki}-67$ protein in human keratinocytes sections in ESWT exposed and untreated controls.

\begin{tabular}{|c|c|c|c|c|}
\hline & \multicolumn{4}{|c|}{ Keratinocytes } \\
\hline & \multicolumn{2}{|c|}{$\begin{array}{c}\text { ESWT } \\
\left(0,1 \mathrm{~mJ} / \mathrm{mm}^{2}, 100 \mathrm{Impulse}, 1\right. \\
\mathrm{Hz}, 5 \mathrm{~cm})\end{array}$} & \multicolumn{2}{|c|}{ Control } \\
\hline & $\begin{array}{c}\text { positivestained } \\
\text { cells }(\%)\end{array}$ & Intensity & $\begin{array}{c}\text { positive stained } \\
\text { cells }(\%)\end{array}$ & Intensity \\
\hline Ki-67 & 14 & ++ to +++ & 10 & ++ to +++ \\
\hline CK 5 & 95 & ++ to +++ & 85 & ++ to +++ \\
\hline CK14 & 90 & ++ & 85 & ++ \\
\hline CK10 & 24 & + to +++ & 33 & + to +++ \\
\hline
\end{tabular}

Table 1: Comparative representation of the expression of cytokeratins CK5, CK14 and CK10 as well as the proliferation marker Ki-67 ESW-treated and untreated keratinocytes as a percentage as well as provision of the intensity of the precipitates formed (legend: $+=$ mild,$++=$ average,$+++=$ strong); $n=5$

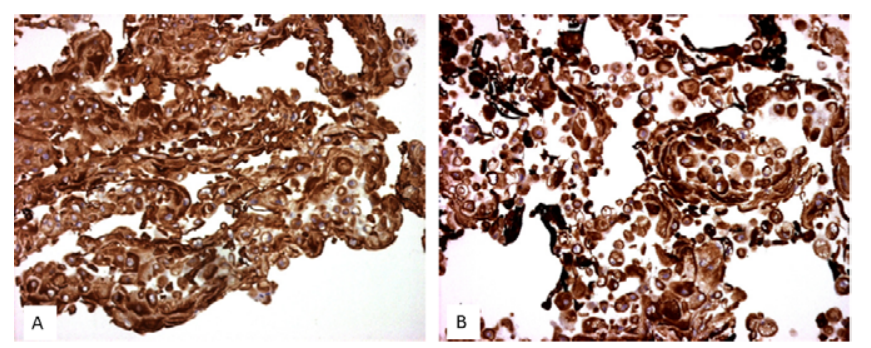

Figure 4: A. ESWT treatment increases number of $C K 5^{+}$keratinocytes in vitro/ Representative IHC image of the CK5 positive human keratinocytes treated with ESWT. B. Untreated control.

10\% amongst the untreated keratinocytes (Figure 3 and Table 1).

ESWT keratinocytes showed increase in the expression of the CK5 and CK14 when compared to the control, untreated keratinocytes. Ninety five per cent of ESWT keratinocytes was positive for CK5 in comparison to $85 \%$ of the untreated controls (Figure 4 and Table 1) and $90 \%$ of the ESWT keratinocytes was positive for CK14 staining in comparison to $85 \%$ of untreated controls (Figure 5 and Table 1). All sections showed moderate to strong intense staining in the different fields. CK10 positive cells were found at frequencies of $24 \%$ in ESWtreated and 33\% in untreated keratinocytes (Figure 6 and Table 1).

\section{Discussion}

Development of novel cultivation techniques is important for the purposes of tissue engineering and development of novel cell-based wound dressings. Keratinocytes play a key role in the repopulation of the skin gaps caused by injury and are essential for the remodelling phase of wound healing. Therefore, successful application of syngenic keratinocytes is no surprise $[14,15]$. Extracorporeal shockwave therapy has also been successfully used clinics for the soft tissue healing, in 


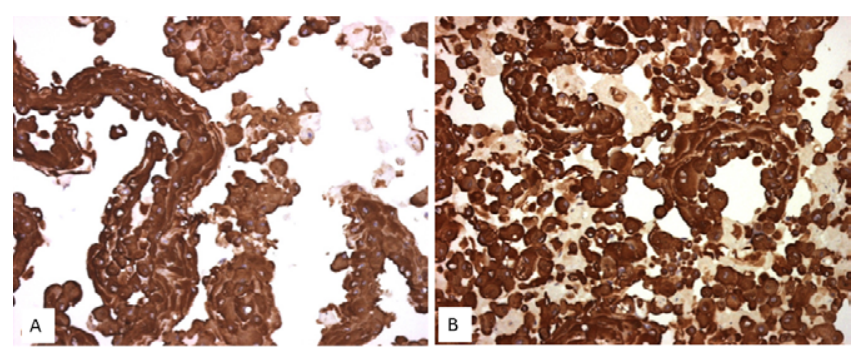

Figure 5: A. Representative image of CK14 positive human keratinocytes treated with ESWT. B. Control.

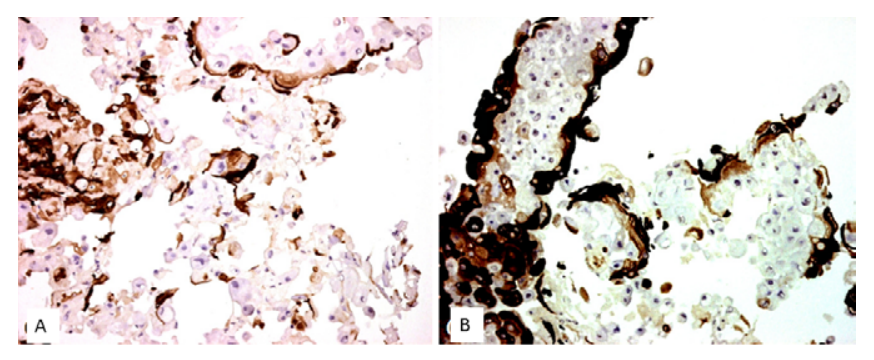

Figure 6: A. Treatment of keratinocytes in vitro with 100 impulses with $E F D=0.1$ $\mathrm{mJ} / \mathrm{mm}^{2}, 1 \mathrm{~Hz}, 5 \mathrm{~cm}$ increases number of $\mathrm{CK} 10^{+}$cells. Representative IHC image of $\mathrm{CK}_{10} 0^{+}$human keratinocytes after exposure to ESWT. B. Untreated controls.

vivo and more recently in vitro, to modify wound healing processes and shifts expression of pro and anti-inflammatory genes and proteins toward successful healing. In vitro, it has been used successfully in fibroblast, osteoblast and tenocyte cultures. In presented study, we sought to determine effect of ESWT on proliferation and differentiation of the primary cultured human keratinocytes and provide insight in the mechanism of ESWT effects in the wound healing process.

Using the proliferation marker Ki-67, 14\% of the cells were found to be actively proliferating in the ESWT keratinocytes, which was $4 \%$ higher than that seen with untreated keratinocytes. This result is consistent with our results (not presented) showing higher count of viable cells after treatment with ESWT. Only basal keratinocytes are capable of dividing into two genetically identical daughter cells. The proliferating keratinocytes can be divided into two groups, namely the 'stem cells' with their highly proliferative potential, and the transit amplifying cells, which can still undergo number of cell division cycles on their way to becoming terminally differentiated. To better understand the effects of ESWT on the keratinocytes in vitro, we evaluated maturation of keratinocytes in shockwave-treated and untreated cultures. To determine changes in cytokeratins we used IHC to detect CK5, CK14 and CK10, the most widely used markers for assessing epithelial differentiation [19]. The cytokeratin pair CK5/14 was expressed predominantly in the basal keratinocytes, while the cytokeratin pair CK1/10 was detected in the post-mitotic suprabasal cell layers. CK1/CK10 are molecules which convey increasing mechanical stability and integrity to the keratinocytes layers [20]. In our study, ESWT keratinocytes have a slightly higher expression of the cytokeratin pair CK5/14, whereas the suprabasal epidermal cytokeratin CK10 was found more in the untreated control keratinocytes. These results suggest a proliferation-enhancing effect of the application of shockwaves, since the proportion of keratinocytes with an expression profile typical of partially or terminally differentiated cells (24\%) was lower than in untreated keratinocytes (33\%). What is interesting in both groups is (ESWT and untreated keratinocytes) that there was an overall high expression of basal cytokeratins with a frequency of 85-95\%, while there was a relatively small proportion of actively proliferating cells $(10-14 \%)$. In the cultured skin, number of hyper proliferating cells is normalized after grafting [20]. This transient nature of proliferative activity in vitro, may explain the relatively small percentage of actively proliferating cells in our experiments. A limitation of the study is the evaluation of only one time point of the dynamic process that is staggered in time over the entire cell population.

The proliferation-enhancing effect of ESW can be explored from different angle. A critical role should also be attributed to a direct stimulatory effect arising from the proliferation inducing effects of hydrodynamic stress. In various experiments, proliferation-promoting effects of shockwave application were demonstrated previously in various cell types $[6,7]$. In addition to a predominantly cytotoxic effect of ESWT at high energy flux densities, increased proliferation at lower energy densities was shown for cultures of fibroblasts, tenocytes, periosteal cells and osteoblasts from human cancellous bone [9,21-23]. With regard to keratinocytes specifically, mechanical stress sensitivity was demonstrated with a growth-promoting effect in strain tests, as well as promotion of differentiation in compression tests [24-26]. In addition, serial time-dependent evaluation of gene expression profiles showed that keratinocytes respond to mechanical stimuli differently after 3 days of mechanical stimulation [27] as well as on frequency of the mechanical pressure changes [28] showing that high frequency pressure changes have higher proliferation reducing effects than low frequency changes. However, whether the pressure component of the shockwave is similar to a mechanical effect of pressure remains an open question that should be answered in comparative studies of various mechanical stimuli on the keratinocytes. In addition, changes in membrane permeability, reported after ESWT, can also unleash a growth-promoting effect of various signal molecules in autocrine or paracrine manner.

In wound healing, the expression of the cytokeratins changes during phase of healing process, and position that keratinocytes have in healing tissue [11]. What this study revealed is that the migrating keratinocytes expressed same, basal cytokeratin. Our results suggest that ESWT might have migration stimulating effects in wound healing serving to accelerate wound closure and completion of re-epithelialization. In our studies, we observed high percentage of basal-like keratinocytes, expressing CK5/CK14 consistent with a higher number of proliferating keratinocytes in the wound edges and wound bed. A recent study in rat full thickness burns, Djedovic et al. showed a significant increase in the number of cell layers in the rat epidermis 5 days after the ESWT treatment. The in vivo investigation of particular populations of the keratinocytes in wound healing of various skin injuries after the ESWT is a goal of future studies.

In conclusion, ESWT of primary keratinocyte cell culture showed increased keratinocyte proliferation. Additionally, ESWT promoted differentiation of the keratinocyte toward basal-layer-like morphology that is known to enhance the rate of wound healing. These promising results merit further investigations of ESWT and non-treated keratinocytes on wound healing in vivo and to provide mechanistic data to support use of ESWT in soft tissue indications.

\section{Acknowledgment}

The MTS Spark Wave ${ }^{\circledR}$ device Dermagold100 (including mandatory annual maintenance and security testing) used in the study was provided by the 
Citation: Antonic V, Hartmann B, Münch S, Belfekroun C, Niedobitek G, et al. (2015) Extracorporeal Shockwaves (ESW) Promote Proliferation and Differentiation of Keratinocytes In vitro-Histology and Immunohistochemistry. J Bioengineer \& Biomedical Sci 5: 161. doi:10.4172/21559538.1000161

Page 5 of 5

manufacturer free of charge. No other funding for the study was given by the manufacturer or its affiliates.

Translation from German into English was carried out by Dr. Julian Keogh (Pharmacad Services, London)

\section{References}

1. Mittermayr R, Antonic V, Hartinger J, Kaufmann H, Redl H, et al. (2012) Extracorporeal shock wave therapy (ESWT) for wound healing: technology, mechanisms, and clinical efficacy. Wound Repair Regen 20: 456-65.

2. Ottomann C, Stojadinovic A, Lavin PT, Gannon FH, Heggeness MH, et al (2012) Prospective randomized phase II Trial of accelerated reepithelialization of superficial second-degree burn wounds using extracorporeal shock wave therapy. Ann Surg 255: 23-29.

3. Ottomann C, Hartmann B, Tyler J, Maier H, Thiele R, et al. (2010) Prospective randomized trial of accelerated re-epithelization of skin graft donor sites using extracorporeal shock wave therapy. J Am Coll Surg 211: 361-367.

4. Zins SR, Amare MF, Tadaki DK, Elster EA, Davis TA (2010) Comparative analysis of angiogenic gene expression in normal and impaired wound healing in diabetic mice: effects of extracorporeal shock wave therapy. Angiogenesis 13: $293-304$

5. Davis TA, Stojadinovic A, Anam K, Amare M, Naik S, et al. (2009) Extracorporea shock wave therapy suppresses the early proinflammatory immune response to a severe cutaneous burn injury. Int Wound J 6: 11-21.

6. Schaden W, Thiele R, Kolpl C, Pusch M, Nissan A, et al. (2007) Shock wave therapy for acute and chronic soft tissue wounds: A feasibility study. J Surg Res 143: 1-12.

7. Schuh CM, Heher P, Weihs AM, Banerjee A, Fuchs C, et al. (2014) In vitro extracorporeal shock wave treatment enhances stemness and preserves multipotency of rat and human adipose- derived stem cells. Cytotherapy 16: 1666-1678.

8. Raabe O, Shell K, Goessl A, Crispens C, Delhasse Y, et al. (2013) Effect of extracorporeal shock wave on proliferation and differentiation of equine adipose tissue-derived mesenchymal stem cells in vitro. Am J Stem Cells 2: 62-73.

9. Berta L, Fazzari A, Ficco AM, Enrica PM, Catalano MG, et al. (2009) Extracorporeal shock waves enhance normal fibroblast proliferation in vitro and activate mRNA expression for TGF-beta1 and for collagen types I and III. Acta Orthop 80: 612-617.

10. Raja, Sivamani K, Garcia MS, Isseroff RR (2007) Wound re-epithelialization: Modulating keratinocyte migration in wound healing. Front Biosci 12: 28492868.

11. Watanabe S, Osumi M, Ohnishi T, Ichikawa E, Takahashi H (1995) Changes in cytokeratin expression in epidermal keratinocytes during wound healing Histochem Cell Biol 103: 425-433.

12. Rasmussen C, Thomas-Virnig C, Allen-Hoffmann BL (2013) Classical human epidermal keratinocyte cell culture. Methods Mol Biol 945: 161-175.
13. Moll R, Divo M, Langbein $L$ (2008) The human keratins: Biology and pathology Histochem Cell Biol 129: 705-733.

14. Fratianne R, Papay F, Housini I, Lang C, Schafer IA (1993) Keratinocyte allografts accelerate healing of split-thickness donor sites: Applications for improved treatment of burns. J Burn Care Rehabil 114: 148-54.

15. Monstrey S, Beele H, Kettler M, Van Landuyt K, Blondeel P, et al.(1999) Allogeneic cultured keratinocytes vs. cadaveric skin to cover wide-mesh autogenous split-thickness skin grafts. Ann Plast Surg 43: 268-72.

16. Scholzen T, Gerdes J (2000) The Ki-67 protein: From the known and the unknown. J Cell Physiol 182: 311-322.

17. Schmidt MH, Broll R, Bruch HP, Duchrow M (2002) Proliferation marker pKi-67 affects the cell cycle in a self-regulated manner. J Cell Biochem 87: 334-341.

18. Gerdes J (1990) Ki-67 and other proliferation markers useful for immunohistological diagnostic and prognostic evaluations in human malignancies. Semin Cancer Biol 1: 199-206.

19. Mischke D, Wild G (1987) Polymorphic keratins in human epidermis. J Invest Dermatol 88: 191-7.

20. Smiley AK, Klingenberg JM, Boyce ST, Supp DM (2006) Keratin expression in cultured skin substitutes suggests that the hyperproliferative phenotype observed in vitro is normalized after grafting. Burns 32: 135-138.

21. Chao YH, Tsuang YH, Sun JS, Chen LT, Chiang YF, et al. (2008) Effects of shock waves on tenocyte proliferation and extracellular matrix metabolism. Ultrasound Med Biol 34:841-52.

22. Tam KF, Cheung WH, Lee KM, Qin L, Leung KS, et al. (2005) Delayed stimulatory effect of low-intensity shockwaves on human periosteal cells. Clin Orthop Relat Res 438:260-5.

23. Hofmann A, Ritz U, Hessmann MH, Alini M, Rommens PM, et al. (2008) Extracorporeal shock wave-mediated changes in proliferation, differentiation, and gene expression of human osteoblasts. J Trauma 65: 1402-10.

24. Silver FH, Siperko LM, Seehra GP (2003) Mechanobiology of force transduction in dermal tissue. Skin Res Technol 9:3-23.

25. Reichelt J (2007) Mechanotransduction of keratinocytes in culture and in the epidermis. Eur J Cell Biol 86: 807-16.

26. Gormar FE, Bernd A, Bereiter-Hahn J, Holzmann H (1990) A new model of epidermal differentiation: Induction by mechanical stimulation. Arch Dermatol Res 282: 22-32.

27. Cherbuin T, Movahednia MM, Toh WS, Cao T (2015) Investigation of human embryonic stem cell-derived keratinocytes as an in vitro research model for mechanical stress dynamic response. Stem Cell Rev 11: 460-473.

28. Nasca MR, Shih AT, West DP, Martinez WM, Micali G (2007) Intermittent pressure decreases human keratinocyte proliferation in vitro. Skin Pharmacol Physiol 20: 305-12. 